\title{
Presentations and Outcomes of Thyroid Storm
}

\author{
Muhammad Shahid1, Zareen Kiran1, Aqiba Sarfraz², Syed Muhammad Hasan1, Syed Muhammad Adnan1 \\ and Akhtar Ali Baloch1 \\ ${ }^{1}$ National Institute of Diabetes \& Endocrinology (NIDE), Dow University of Health Sciences (Ojha Campus) Karachi, Pakistan \\ ${ }^{2}$ Liaquat National Hospital \& Medical College Karachi, Pakistan
}

\begin{abstract}
The aim of this study is to describe the characteristics and outcome of thyroid storm patients presenting at two tertiary care centres of Karachi, i.e. at Dow University of Health Sciences; and Liaquat National Hospital, Karachi, from December 2018 to May 2019. All patients between 18-70 years of age, who were admitted with thyroid storm and fulfilled the BurchWartofsky criteria, were inducted. Demographics including age, gender, clinical presentations, systemic symptoms, clinical examination of the thyroid and laboratory findings were reviewed from the file records to identify factors associated with mortality using the available data. Overall, five (62.5\%) out of eight patients were female. The mean age was $43 \pm 1.67$ years. Infections were the most common comorbid condition followed by cardiovascular and gastrointestinal diseases. The in-hospital mortality rate was $87.5 \%(n=07)$.
\end{abstract}

Key Words: Thyrotoxicosis, Thyroid storm, Outcome, Emergency.

How to cite this article: Shahid M, Kiran Z, Sarfraz A, Hasan SM, Adnan SM, Baloch AA. Presentations and outcomes of thyroid storm. $J$ Coll Physicians Surg Pak 2020; 30(3):330-331.

Thyroid storm (TS) is a life-threatening endocrine emergency. It is one of the dreaded complications of an untreated or improperly treated common thyroid disorder called Graves' disease. 1 However, there are cases with untreated multinodular goiter, which were presented with thyroid storm as well. 2 It is known to occur after a precipitating event like an ischemic insult, or an intercurrent illness like infection or septicemia. Clinical features encompass a multitude of systems' manifes-tations and include high body temperature, and tachycardia: particularly atrial fibrillation, altered sensorium and gastroenterological hyperactivity. ${ }^{3}$ The diagnosis is made on high clinical suspicion and is often based on one of the internationally accepted criteria; for example, BurchWartofsky scale (BWS). ${ }^{4}$ According to BWS, a score of 45 or more out of 140 is highly suggestive of thyroid storm; 25-44 out of 140 suggest impending; whereas, a score of less than 25 does not have thyroid storm.

This study was approved by both Institutes' Ethical Review Committees prior to initiation of the research work. A retrospective longitudinal study was conducted in National Institute of Diabetes and Endocrinology (NIDE), Dow University of Health Sciences (DUHS), Ojha Campus; and the Section of Endocrinology, Department of Medicine, Liaquat National Hospital (LNH), Karachi.

Correspondence to: Dr. Muhammad Shahid, National Institute of Diabetes \& Endocrinology (NIDE), Dow University of Health Sciences (Ojha Campus), Karachi, Pakistan

E-mail: mohd_shahid72@yahoo.com

Received: September 24, 2019; Revised: December 23, 2019; Accepted: January 14, 2020
All patients between 18-70 years of age, who were admitted with thyroid storm and fulfilled BWS criteria, were included in the study. Pregnant females and those with psychiatric illnesses were excluded. Demographics including age, gender, clinical presentations, systemic symptoms, and clinical examination of the thyroid were reviewed from the file records to identify factors associated with mortality using the available data. Five cases from DUHS and three from LNH were taken. BWS scoring was calculated in each patient. Signs and symptoms, laboratory investigations including complete blood count (CBC), thyroid stimulating hormone (TSH), free thyroxine (FT4), free triiodothyronine (FT3), Liver Function Test (LFT), were reviewed through detailed proforma. Descriptive statistics were performed on the collected socio-demographic characteristics, clinical features, laboratory findings, treatment modalities, and patient outcomes by calculating percentages. All multinodular goiter and Graves' disease patients were biochemically thyrotoxic at the time of thyroid storm presentation.

Overall, $5(62.5 \%)$ of the total eight patients were females. The mean age of the patients was $43 \pm 1.67$ years. Winter season was associated with highest number of thyroid storm. Seven patients required ventilatory support with a similar rate of in-hospital mortality. Three cases were newly diagnosed. Infections (mostly respiratory) were the most common comorbid condition, followed by cardiovascular and gastrointestinal diseases. Six (75\%) cases were already diagnosed with Graves' disease. However, none of them were taking anti-thyroid medications at the time of admission. Two (25\%) had 
Table I: Clinical findings of thyroid storm patients

\begin{tabular}{|c|c|c|c|c|c|c|c|c|c|c|c|}
\hline Patients & $\begin{array}{l}\text { Age } \\
\text { (years) }\end{array}$ & Diagnosis & $\begin{array}{l}\text { Comorbidities/ } \\
\text { precipitating events }\end{array}$ & Gender & $\begin{array}{c}\text { Blood } \\
\text { pressure } \\
(\mathrm{mmHg})\end{array}$ & $\begin{array}{c}\text { Pulse } \\
\text { (beats/min) }\end{array}$ & Temp & $\begin{array}{c}\text { Oxygen } \\
(\%)\end{array}$ & GCS & BWS & $\begin{array}{c}\text { Duration } \\
\text { of hospital } \\
\text { stay }\end{array}$ \\
\hline 1 & 21 & Graves disease & Lower respiratory infection & Female & $120 / 76$ & 110 & 100 & 96 & $\leq 8$ & $76-100$ & 7 days \\
\hline 2 & 50 & Multinodular goiter & Gastrointestinl infection & Female & $140 / 90$ & 132 & 99 & 92 & $\leq 8$ & $76-100$ & 6 days \\
\hline 3 & 60 & Multinodular goiter & Urinary tract infection & Female & $150 / 90$ & 140 & 102 & 98 & $9-14$ & $76-100$ & 4 days \\
\hline 4 & 47 & Graves disease & Lower respiratory tract infection & Male & $110 / 70$ & 110 & 99 & 97 & $9-14$ & $45-75$ & 7 days \\
\hline 5 & 32 & Gravesdisease & $\begin{array}{l}\text { Cardiovascular disease } \\
\text { (heart failure) }\end{array}$ & Male & $120 / 60$ & 120 & 102 & 97 & $9-14$ & $45-75$ & 4 days \\
\hline 6 & 20 & Gravesdisease & Urinary tract infection & Female & $100 / 60$ & 130 & 101 & 97 & $9-14$ & $45-75$ & 8 days \\
\hline 7 & 60 & Gravesdisease & Respiratory failure & Female & $110 / 70$ & 125 & 102 & 94 & $9-14$ & $45-75$ & 10 days \\
\hline 8 & 57 & Gravesdisease & Sepsis & Male & $110 / 65$ & 130 & 101 & 97 & $9-14$ & $45-75$ & 9 days \\
\hline
\end{tabular}

multinodular goiter. Most patients with thyroid storm presented with BWS criteria in the range of 76-100 (5 cases- $62.5 \%$ ). Glasgow coma scale (GCS) of these patients was scored $9-14$ in $6(75 \%)$ cases. Seven $(87.5 \%)$ patients had tachycardia.

All patients were anemic; one was severely anemic (Hemoglobin $3.4 \mathrm{~g} / \mathrm{dl}$ ). Leucocyte count was raised in all patients, except one who had leucopenia. Three out of eight patients had hepatitis biochemical picture of liver function tests, but all of them had raised serum bilirubin levels. Estimated GFR (glomerular filtration rate) was decreased in only Two (25\%) cases (47.22 and 56.67 $\mathrm{ml} / \mathrm{min}$ ). Precipitating events of thyroid storm in eight patients are given in Table I. These comorbidities had aggravated the underlying hyperthyroidism and had led to the high mortality of these patients. Five $(62.5 \%)$ patients were treated with methimazole and $1(12.5 \%)$ with propylthiouracil. However, all patients received steroids.

There was high mortality in this study as compared to the West. 5 There can be several reasons for this difference, as the main limitation of the study is that the number of observed cases is very small; whereas, these studies are based on large cohorts and national database. Besides, further prospective studies to determine whether the cause of high mortality is infections or other underlying condition instead of the thyroid storm. Most of these cases had infections on admission as a precipitating cause. Our part of the world has more communicable diseases as compared to the rest of the world, which can be one reason of higher prevalence of respiratory tract, gastrointestinal and urinary tract infections. Further, the issue of compliance with antithyroid medications remains an important factor to be addressed. Two cases had multinodular goiter, out of which one was also previously reported as a case report. ${ }^{2}$

All these patients were admitted through emergency department. Most of these patients had some sort of infections and required hydration, intravenous antibiotics, and steroids as part of initial management of their underlying disease. It takes some time to establish the actual diagnosis based on BWS through investigations including thyroid profile, especially in undiagnosed thyroid storm cases. During the initial phase of management, it is appropriate to manage these patients as having underlying sepsis, but keeping high threshold for thyroid storm. Once investigations are completed, heart rate should be controlled with betablockers and definitive treatment be initiated with no further delay.

Due to limited resources, some investigations were not done like inflammatory markers and TSH receptor stimulating antibodies. The authors recommend more prospective longitudinal studies in collaboration with emergency department physicians, intensivists, internists and endocrinologists to reflect the true burden of this disease and under recognised endocrine emergency.

\section{CONFLICT OF INTEREST:}

Authors declared no conflict of interest.

\section{AUTHORS' CONTRIBUTION:}

MS, ZK, AS: Written the protocol and taken IRB approval, gathered the data, final article writeup.

MS, ZK, AS, SMH: Gathered the data, final article writeup.

SMA: Did the statistical analysis.

AAB: Final article writeup.

\section{REFERENCES}

1. Chiha M, Samarasinghe S, Kabaker AS. Thyroid storm: An updated review. J Intensive care Med 2015; 30:131-40.

2. Shahid M, Mahar SA, Asghar A, Alam M. Thyroid storm associated with multinodular goiter: A difficult problem to treat. J Coll Physicians Surg Pak 2015; 25:225-6.

3. Carroll R, Matfin G. Endocrine and metabolic emergencies: Thyroid storm. Ther Adv Endocrinol Metab 2010; 1:139-45.

4. Burch HB, Wartofsky L. Life-threatening thyrotoxicosis: Thyroid storm. Endocrinol Metab Clin North Am 1993; 22:263-77.

5. Angell TE, Lechner MG, Nguyen CT, Salvato VL, Nicoloff JT, LoPresti JS. Clinical features and hospital outcomes in thyroid storm: A retrospective cohort study. J Clin Endocrinol Metab 2015; 100:451-9. 